Research Article

\title{
Global Attractivity in a Discrete Mutualism Model with Infinite Deviating Arguments
}

\author{
Xiangdong Xie, ${ }^{1}$ Yalong Xue, ${ }^{1}$ and Runxin $\mathrm{Wu}^{2}$ \\ ${ }^{1}$ Department of Mathematics, Ningde Normal University, Ningde, Fujian 352300, China \\ ${ }^{2}$ College of Mathematics and Physics, Fujian University of Technology, Fuzhou, Fujian 350014, China
}

Correspondence should be addressed to Xiangdong Xie; ndsyxxd@163.com

Received 17 August 2016; Accepted 14 November 2016; Published 12 February 2017

Academic Editor: Garyfalos Papashinopoulos

Copyright (C) 2017 Xiangdong Xie et al. This is an open access article distributed under the Creative Commons Attribution License, which permits unrestricted use, distribution, and reproduction in any medium, provided the original work is properly cited.

A set of sufficient conditions is obtained for the global attractivity of the following two-species discrete mutualism model with infinite deviating arguments: $x_{1}(k+1)=x_{1}(k) \exp \left\{r_{1}\left[\left(K_{1}+\alpha_{1} \sum_{s=0}^{+\infty} J_{2}(s) x_{2}(k-s)\right) /\left(1+\sum_{s=0}^{+\infty} J_{2}(s) x_{2}(k-s)\right)-x_{1}(k)\right]\right\}$ and $x_{2}(k+1)=$ $x_{2}(k) \exp \left\{r_{2}\left[\left(K_{2}+\alpha_{2} \sum_{s=0}^{+\infty} J_{1}(s) x_{1}(k-s)\right) /\left(1+\sum_{s=0}^{+\infty} J_{1}(s) x_{1}(k-s)\right)-x_{2}(k)\right]\right\}$, where $r_{i}, K_{i}, \alpha_{i}, i=1,2$, are all positive constants, $\sum_{j=1}^{+\infty} J_{i}(n)=1$, and $\alpha_{i}>K_{i}$. Our results generalize the main result of Yang et al. (2014).

\section{Introduction}

The aim of this paper is to investigate the stability property of the following two-species discrete mutualism model with infinite deviating arguments:

$$
\begin{aligned}
& x_{1}(k+1)=x_{1}(k) \\
& \cdot \exp \left\{r_{1}\left[\frac{K_{1}+\alpha_{1} \sum_{s=0}^{+\infty} J_{2}(s) x_{2}(k-s)}{1+\sum_{s=0}^{+\infty} J_{2}(s) x_{2}(k-s)}-x_{1}(k)\right]\right\}, \\
& x_{2}(k+1)=x_{2}(k) \\
& \cdot \exp \left\{r_{2}\left[\frac{K_{2}+\alpha_{2} \sum_{s=0}^{+\infty} J_{1}(s) x_{1}(k-s)}{1+\sum_{s=0}^{+\infty} J_{1}(s) x_{1}(k-s)}-x_{2}(k)\right]\right\},
\end{aligned}
$$

together with the initial conditions

$$
\begin{aligned}
& x_{i}(s)=\phi_{i}(s) \geq 0, \\
& \quad x_{i}(0)>0, s=\cdots,-k,-k+1, \ldots,-2,-1, i=1,2 .
\end{aligned}
$$

$\mathrm{Li}$ and $\mathrm{Xu}$ [1] studied the following two-species integrodifferential model of mutualism:

$$
\begin{aligned}
N_{1}^{\prime}(t) & =r_{1}(t) N_{1}(t) \\
\cdot & {\left[\frac{K_{1}(t)+\alpha_{1}(t) \int_{0}^{\infty} J_{2}(s) N_{2}(t-s) d s}{1+\int_{0}^{\infty} J_{2}(s) N_{2}(t-s) d s}\right.} \\
- & \left.N_{1}\left(t-\sigma_{1}(t)\right)\right], \\
N_{2}^{\prime}(t) & =r_{2}(t) N_{2}(t) \\
\cdot & {\left[\frac{K_{2}(t)+\alpha_{2}(t) \int_{0}^{\infty} J_{1}(s) N_{1}(t-s) d s}{1+\int_{0}^{\infty} J_{1}(s) N_{1}(t-s) d s}\right.} \\
& \left.-N_{2}\left(t-\sigma_{2}(t)\right)\right] .
\end{aligned}
$$

Under the assumption $K_{i}(t), \alpha_{i}(t), i=1,2$, are all positive periodic functions and $\alpha_{i}>K_{i}, i=1,2$, by applying the coincidence degree theory, they showed that system (3) admits at least one positive $\omega$-periodic solution. Chen and You [2] 
argued that a general nonautonomous nonperiodic system is more appropriate, and for the general nonautonomous case, by using the differential inequality theory, they showed that the system is permanent. It brings to our attention that both $[1,2]$ did not consider the stability property of the system, and in [3], under the assumption $r_{i}, K_{i}, \alpha_{i}, i=1,2$, are all positive constants, $\sigma_{i}(t) \equiv 0$, we investigated the stability property of the system, and we showed that the system admits a unique globally attractive positive equilibrium. At the end of the paper, we pointed out "whether some parallel result could be established for the discrete type mutualism system is still unknown, we leave this for future investigation."

Previously, corresponding to system (3), Li and Yang [4] and $\mathrm{Li}$ [5] proposed the following two-species discrete model of mutualism with infinite deviating arguments:

$$
\begin{array}{r}
x_{1}(k+1)=x_{1}(k) \exp \left\{r_{1}(k)\right. \\
\cdot\left[\frac{K_{1}(k)+\alpha_{1}(k) \sum_{s=0}^{+\infty} J_{2}(s) x_{2}(k-s)}{1+\sum_{s=0}^{+\infty} J_{2}(s) x_{2}(k-s)}\right. \\
\left.\left.-x_{1}\left(k-\delta_{1}(k)\right)\right]\right\}, \\
x_{2}(k+1)=x_{2}(k) \exp \left\{r_{2}(k)\right. \\
\cdot\left[\frac{K_{2}(k)+\alpha_{2}(k) \sum_{s=0}^{+\infty} J_{1}(s) x_{1}(k-s)}{1+\sum_{s=0}^{+\infty} J_{1}(s) x_{1}(k-s)}\right. \\
\left.\left.-x_{2}\left(k-\delta_{2}(k)\right)\right]\right\},
\end{array}
$$

where $x_{i}(k), i=1,2$, is the density of mutualism species $i$ at the $k$ th generation and $\left\{r_{i}(k)\right\},\left\{K_{i}(k)\right\},\left\{\alpha_{i}(k)\right\},\left\{J_{i}(k)\right\}$, and $\left\{\delta_{i}(k)\right\}, i=1,2$, are bounded nonnegative sequences such that

$$
\begin{aligned}
& 0<r_{i}^{l} \leq r_{i}^{u}, \\
& 0<a_{i}^{l} \leq a_{i}^{u}, \\
& 0<K_{i}^{l} \leq K_{i}^{u}, \\
& 0<\delta_{i}^{l} \leq \delta_{i}^{u}, \\
& \sum_{j=1}^{+\infty} J_{i}(n)=1, \\
& \alpha_{i}>K_{i} .
\end{aligned}
$$

They showed that, under the above assumption, system (4) is permanent. Again, none of the papers $[4,5]$ considered the stability property of the system. To make an intensive study on this direction, in [6], we investigated the dynamic behaviors of the following autonomous mutualism system:

$$
\begin{aligned}
& x_{1}(k+1) \\
& \quad=x_{1}(k) \exp \left\{r_{1}\left[\frac{K_{1}+\alpha_{1} x_{2}(k)}{1+x_{2}(k)}-x_{1}(k)\right]\right\}, \\
& x_{2}(k+1) \\
& \quad=x_{2}(k) \exp \left\{r_{2}\left[\frac{K_{2}+\alpha_{2} x_{1}(k)}{1+x_{1}(k)}-x_{2}(k)\right]\right\},
\end{aligned}
$$

where $x_{i}(k)(i=1,2)$ are the population density of the $i$ th species at $k$-generation. We showed that if

$$
\begin{aligned}
& \left(H_{1}\right) r_{i}, K_{i}, \alpha_{i}(i=1,2) \text { are all positive constants and } \alpha_{i}> \\
& K_{i}(i=1,2) \\
& \left(H_{2}\right) r_{i} \alpha_{i} \leq 1,(i=1,2)
\end{aligned}
$$

hold, system (6) admits a unique positive equilibrium $\left(x_{1}^{*}\right.$, $x_{2}^{*}$ ), which is globally asymptotically stable. Our result shows that the dynamic behavior of the discrete type mutualism model is more complicated, and one could not expect to establish parallel result as that of continuous ones. Also, at the end of the paper, we pointed out "it seems interesting to incorporate the time delay to the system (6) and investigate the dynamic behaviors of the system, we leave this for future study." However, to this day, we still did not study the correspondence topic on this area. For more background of system (3), (4), and (6) one could refer to [1-24] and the references cited therein. We mention here that, with $\sigma_{i}(k) \neq 0, i=1,2$, and all the coefficients being time-dependent, system (4) is a nonautonomous pure-delay system, and it is not an easy thing to investigate the stability property of the system. This motivated us to discuss the simple one, that is, the autonomous simple non-pure-delay system (1).

Concerned with the stability property of system (1)-(2), we have the following result.

Theorem 1. Assume that $\left(H_{1}\right)$ and $\left(H_{2}\right)$ hold, and then system (1)-(2) admits a unique positive equilibrium $\left(x_{1}^{*}, x_{2}^{*}\right)$, which is globally attractive.

Remark 2. Obviously, Theorem 1 generalizes the main results of Yang et al. [6] to the infinite deviating arguments case. Theorem 1 can also be seen as the parallel result of the continuous one in [3]. Thus, we push on the study of the mutualism model.

\section{Existence and Uniqueness of Positive Equilibrium}

This section focuses on the existence and uniqueness of positive equilibrium of system (1). More precisely, we will prove the following result.

Theorem 3. Under the assumption of Theorem 1, system (1)-(2) admits a unique positive equilibrium. 
Proof. The positive equilibrium of system (1) satisfies

$$
\begin{aligned}
& \frac{K_{1}+\alpha_{1} x_{2}}{1+x_{2}}-x_{1}=0, \\
& \frac{K_{2}+\alpha_{2} x_{1}}{1+x_{1}}-x_{2}=0,
\end{aligned}
$$

which is equivalent to

$$
\begin{gathered}
A_{1} x_{1}^{2}+A_{2} x_{1}+A_{3}=0, \\
B_{1} x_{2}^{2}+B_{2} x_{2}+B_{3}=0,
\end{gathered}
$$

where

$$
\begin{aligned}
& A_{1}=\alpha_{2}+1, \\
& A_{2}=-\alpha_{1} \alpha_{2}-K_{1}+K_{2}+1, \\
& A_{3}=-K_{2} \alpha_{1}-K_{1}, \\
& B_{1}=\alpha_{1}+1, \\
& B_{2}=-\alpha_{1} \alpha_{2}+K_{1}-K_{2}+1, \\
& B_{3}=-K_{1} \alpha_{2}-K_{2} .
\end{aligned}
$$

Now let us consider the function

$$
F_{1}\left(x_{1}\right)=A_{1} x_{1}^{2}+A_{2} x_{1}+A_{3}
$$

and since $A_{1}>0, A_{3}<0$, it follows that $F_{1}(-\infty)=$ $+\infty, F_{1}(0)=0, F_{1}(+\infty)=+\infty$, and, hence, from the continuity of $F_{1}$, there exist two points $x_{1}^{* *}$ and $x_{1}^{*}, x_{1}^{* *}<$ $0<x_{1}^{*}$, such that $F_{1}\left(x_{1}^{* *}\right)=F_{1}\left(x_{1}^{*}\right)=0$, and since $F_{1}\left(x_{1}\right)=0$ has at most two solutions, it means that $F_{1}\left(x_{1}\right)=0$ admits unique positive solution $x_{1}^{*}$. Similarly, from $B_{1}>0, B_{3}<0$, one could prove $F_{2}\left(x_{2}\right)=B_{1} x_{2}^{2}+B_{2} x_{2}+B_{3}=0$ admits unique positive solution $x_{2}^{*}$. By simple computation, system (7) admits a unique positive solution $E_{+}\left(x_{1}^{*}, x_{2}^{*}\right)$, where

$$
\begin{aligned}
& x_{1}^{*}=\frac{-A_{2}+\sqrt{A_{2}^{2}-4 A_{1} A_{3}}}{2 A_{1}}, \\
& x_{2}^{*}=\frac{-B_{2}+\sqrt{B_{2}^{2}-4 B_{1} B_{3}}}{2 B_{1}} .
\end{aligned}
$$

This ends the proof of Theorem 3.

\section{Proof of Theorem 1}

Now we state several lemmas which will be useful in the proof of Theorem 1 .

Lemma 4 (see [25]). Let $f(u)=u \exp (\alpha-\beta u)$, where $\alpha$ and $\beta$ are positive constants, and then $f(u)$ is nondecreasing for $u \in$ $(0,1 / \beta]$.
Lemma 5 (see [25]). Assume that sequence $\{u(k)\}$ satisfies

$$
u(k+1)=u(k) \exp (\alpha-\beta u(k)), \quad k=1,2, \ldots,
$$

where $\alpha$ and $\beta$ are positive constants and $u(0)>0$. Then

(i) If $\alpha<2$, then $\lim _{k \rightarrow+\infty} u(k)=\alpha / \beta$.

(ii) If $\alpha \leq 1$, then $u(k) \leq 1 / \beta, k=2,3, \ldots$.

Lemma 6 (see [26]). Suppose that functions $f, g: Z_{+} \times$ $[0, \infty) \rightarrow[0, \infty)$ satisfy $f(k, x) \leq g(k, x)(f(k, x) \geq g(k, x))$ for $k \in Z_{+}$and $x \in[0, \infty)$ and $g(k, x)$ is nondecreasing with respect to $x$. If $\{x(k)\}$ and $\{u(k)\}$ are the nonnegative solutions of the following difference equations:

$$
\begin{aligned}
& x(k+1)=f(k, x(k)), \\
& u(k+1)=g(k, u(k)),
\end{aligned}
$$

respectively, and $x(0) \leq u(0)(x(0) \geq u(0))$, then

$$
x(k) \leq u(k)(x(k) \geq u(k)), \quad \forall k \geq 0 .
$$

Lemma 7 (see [27]). Let $x: Z \rightarrow R$ be nonnegative bounded sequences, and let $H: N \rightarrow R$ be nonnegative sequences such that $\sum_{n=0}^{\infty} H(n)=1$. Then

$$
\begin{aligned}
\liminf _{n \rightarrow+\infty} x(n) & \leq \liminf _{n \rightarrow+\infty} \sum_{s=-\infty}^{n} H(n-s) x(s) \\
& \leq \limsup _{n \rightarrow+\infty} \sum_{s=-\infty}^{n} H(n-s) x(s) \\
& \leq \limsup _{n \rightarrow+\infty} x(n)
\end{aligned}
$$

Lemma 8. Let $g_{i}(x)=\left(K_{i}+\alpha_{i} x\right) /(1+x), i=1,2$, assume that $\alpha_{i}>K_{i}$, and then $g_{i}(x)$ are the strictly increasing function of $x$.

Proof. Since

$$
g_{i}^{\prime}(x)=-\frac{\left(K_{i}-\alpha_{i}\right)}{(1+x)^{2}}>0, \quad i=1,2,
$$

the conclusion of Lemma 8 immediately follows.

Now we are in the position to prove the main result of this paper.

Proof of Theorem 1. Let $\left(x_{1}(k), x_{2}(k)\right)$ be arbitrary solution of system (1) with initial condition (2). Denote

$$
\begin{aligned}
& U_{i}=\limsup _{k \rightarrow+\infty} x_{i}(k), \\
& V_{i}=\liminf _{k \rightarrow+\infty} x_{i}(k), \\
& \qquad i=1,2 .
\end{aligned}
$$

We claim that $U_{1}=V_{1}=x_{1}^{*}$ and $U_{2}=V_{2}=x_{2}^{*}$. 
From the first equation of system (1), we obtain

$$
\begin{aligned}
& x_{1}(k+1)=x_{1}(k) \\
& \cdot \exp \left\{r _ { 1 } \left[\frac{K_{1}+\alpha_{1} \sum_{s=0}^{+\infty} J_{2}(s) x_{2}(k-s)}{1+\sum_{s=0}^{+\infty} J_{2}(s) x_{2}(k-s)}\right.\right. \\
& \left.\left.-x_{1}(k)\right]\right\} \leq x_{1}(k) \\
& \cdot \exp \left\{r _ { 1 } \left[\frac{\alpha_{1}+\alpha_{1} \sum_{s=0}^{+\infty} J_{2}(s) x_{2}(k-s)}{1+\sum_{s=0}^{+\infty} J_{2}(s) x_{2}(k-s)}\right.\right. \\
& \left.\left.-x_{1}(k)\right]\right\} \leq x_{1}(k) \exp \left\{r_{1} \alpha_{1}-r_{1} x_{1}(k)\right\}, \\
& k=0,1,2, \ldots,
\end{aligned}
$$

considering the auxiliary equation as follows:

$$
\begin{aligned}
& u(k+1)=u(k) \exp \left\{r_{1} \alpha_{1}-r_{1} u(k)\right\}, \\
& \qquad k=0,1,2, \ldots .
\end{aligned}
$$

Because of $0<r_{1} \alpha_{1} \leq 1$, according to (ii) of Lemma 5, we can obtain $u(k) \leq 1 / r_{1}$ for all $k \geq 2$, where $u(k)$ is arbitrary positive solution of (18) with initial value $u(0)>0$. From Lemma $4, f(u)=u \exp \left(r_{1} \alpha_{1}-r_{1} u\right)$ is nondecreasing for $u \in$ $\left(0,1 / r_{1}\right]$. According to Lemma 6 we can obtain $x_{1}(k) \leq u(k)$ for all $k \geq 2$, where $u(k)$ is the solution of (19) with the initial value $u(2)=x_{1}(2)$. According to (i) of Lemma 5 , we can obtain

$$
U_{1}=\limsup _{k \rightarrow+\infty} x_{1}(k) \leq \lim _{k \rightarrow+\infty} u(k)=\alpha_{1}
$$

From (20) and Lemma 7 we have

$$
\begin{aligned}
& \limsup _{k \rightarrow+\infty} \sum_{s=0}^{+\infty} J_{1}(s) x_{1}(k-s) \\
& \quad=\limsup _{n \rightarrow+\infty} \sum_{s=-\infty}^{k} J_{1}(k-s) x_{1}(s) \leq \limsup _{k \rightarrow+\infty} x_{1}(k) \\
& \quad \leq \alpha_{1} .
\end{aligned}
$$

From the second equation of system (1), we obtain

$$
\begin{aligned}
x_{2}(k+1) \leq x_{2}(k) \exp \left\{r_{2} \alpha_{2}-r_{2} x_{2}(k)\right\}, & \\
\qquad k & =0,1,2, \ldots .
\end{aligned}
$$

Similar to the above analysis, we have

$$
U_{2}=\limsup _{k \rightarrow+\infty} x_{2}(k) \leq \alpha_{2} .
$$

From (23) and Lemma 7 we have

$$
\begin{aligned}
& \limsup _{k \rightarrow+\infty} \sum_{s=0}^{+\infty} J_{2}(s) x_{2}(k-s) \\
& \quad=\limsup _{n \rightarrow+\infty} \sum_{s=-\infty}^{k} J_{2}(k-s) x_{2}(s)=\underset{k \rightarrow+\infty}{\limsup } x_{2}(k) \\
& \leq \alpha_{2} .
\end{aligned}
$$

For $\varepsilon>0$ enough small, without loss of generality, we may assume that $\varepsilon<(1 / 2) \min \left\{K_{1}, K_{2}\right\}$, and it follows from (20)(24) that there is an integer $k_{1}>2$ such that, for all $k>k_{1}$,

$$
\begin{array}{r}
x_{1}(k)<\alpha_{1}+\varepsilon \stackrel{\text { def }}{=} M_{1}^{x_{1}}, \\
x_{2}(k)<\alpha_{2}+\varepsilon \stackrel{\text { def }}{=} M_{1}^{x_{2},} \\
\sum_{s=0}^{+\infty} J_{1}(s) x_{1}(k-s)<\alpha_{1}+\varepsilon \stackrel{\text { def }}{=} M_{1}^{x_{1}}, \\
\sum_{s=0}^{+\infty} J_{2}(s) x_{2}(k-s)<\alpha_{2}+\varepsilon \stackrel{\text { def }}{=} M_{1}^{x_{2}} .
\end{array}
$$

For $k \geq k_{1}$, according to the first equation of system (1) we can obtain

$$
\begin{aligned}
& x_{1}(k+1)=x_{1}(k) \\
& \cdot \exp \left\{r _ { 1 } \left[\frac{K_{1}+\alpha_{1} \sum_{s=0}^{+\infty} J_{2}(s) x_{2}(k-s)}{1+\sum_{s=0}^{+\infty} J_{2}(s) x_{2}(k-s)}\right.\right. \\
& \left.\left.-x_{1}(k)\right]\right\} \geq x_{1}(k) \\
& \cdot \exp \left\{r _ { 1 } \left[\frac{K_{1}+K_{1} \sum_{s=0}^{+\infty} J_{2}(s) x_{2}(k-s)}{1+\sum_{s=0}^{+\infty} J_{2}(s) x_{2}(k-s)}\right.\right. \\
& \left.\left.-x_{1}(k)\right]\right\} \geq x_{1}(k) \exp \left\{r_{1} K_{1}-r_{1} x_{1}(k)\right\},
\end{aligned}
$$

considering the auxiliary equation as follows:

$$
u(k+1)=u(k) \exp \left\{r_{1} K_{1}-r_{1} u(k)\right\}
$$

According to (ii) of Lemma 5, we can obtain $u(k) \leq 1 / r_{1}$ for all $k \geq k_{2}$, where $u(k)$ is arbitrary positive solution of (28) with initial value $u\left(k_{2}\right)>0$. From Lemma 4, $f(u)=u \exp \left(r_{1} K_{1}-r_{1} u\right)$ is nondecreasing for $u \in\left(0,1 / r_{1}\right]$. According to Lemma 6 we can obtain $x_{1}(k) \geq u(k)$ for all $k \geq 2$, where $u(k)$ is the solution of (28) with the initial value $u\left(k_{2}\right)=x_{1}\left(k_{2}\right)$. According to (i) of Lemma 5, we have

$$
V_{1}=\liminf _{k \rightarrow+\infty} x_{1}(k) \geq \lim _{k \rightarrow+\infty} u(k)=K_{1} .
$$

From (29) and Lemma 7 we can obtain

$$
\liminf _{k \rightarrow+\infty} \sum_{s=0}^{+\infty} J_{1}(s) x_{1}(k-s) \geq \liminf _{k \rightarrow+\infty} x_{1}(k) \geq K_{1} .
$$

From the second equation of system (1), we obtain

$$
x_{2}(k+1) \geq x_{2}(k) \exp \left\{r_{2} K_{2}-r_{2} x_{2}(k)\right\} .
$$

Similar to the analysis of (27)-(30), we have

$$
V_{2}=\liminf _{k \rightarrow+\infty} x_{2}(k) \geq K_{2},
$$

$$
\liminf _{k \rightarrow+\infty} \sum_{s=0}^{+\infty} J_{2}(s) x_{2}(k-s) \geq K_{2} \text {. }
$$


Then, for the above $\varepsilon>0$, there is an integer $k_{2}>k_{1}$ such that, for all $k>k_{2}$,

$$
\begin{array}{r}
x_{1}(k)>K_{1}-\varepsilon \stackrel{\text { def }}{=} m_{1}^{x_{1}}, \\
x_{2}(k)>K_{2}-\varepsilon \stackrel{\text { def }}{=} m_{1}^{x_{2}} ; \\
\sum_{s=0}^{+\infty} J_{1}(s) x_{1}(k-s)>K_{1}-\varepsilon \stackrel{\text { def }}{=} m_{1}^{x_{1}}, \\
\sum_{s=0}^{+\infty} J_{2}(s) x_{2}(k-s)>K_{2}-\varepsilon \stackrel{\text { def }}{=} m_{1}^{x_{2}} .
\end{array}
$$

Noting that, from Lemma $8, g_{i}(x)=\left(K_{i}+\alpha_{i} x\right) /(1+x)\left(\alpha_{i}>\right.$ $K_{i}$ ) is a strictly increasing function, then, from the first and second equations of system (1) and (26), we have

$$
\begin{aligned}
& x_{1}(k+1)=x_{1}(k) \\
& \cdot \exp \left\{r _ { 1 } \left[\frac{K_{1}+\alpha_{1} \sum_{s=0}^{+\infty} J_{2}(s) x_{2}(k-s)}{1+\sum_{s=0}^{+\infty} J_{2}(s) x_{2}(k-s)}\right.\right. \\
& \left.\left.-x_{1}(k)\right]\right\} \leq x_{1}(k) \exp \left\{r _ { 1 } \left[\frac{K_{1}+\alpha_{1} M_{1}^{x_{2}}}{1+M_{1}^{x_{2}}}\right.\right. \\
& \left.\left.-x_{1}(k)\right]\right\}, \\
& x_{2}(k+1)=x_{2}(k) \\
& \cdot \exp \left\{r _ { 2 } \left[\frac{K_{2}+\alpha_{1} \sum_{s=0}^{+\infty} J_{1}(s) x_{1}(k-s)}{1+\sum_{s=0}^{+\infty} J_{1}(s) x_{1}(k-s)}\right.\right. \\
& \left.\left.-x_{2}(k)\right]\right\} \leq x_{2}(k) \exp \left\{r _ { 2 } \left[\frac{K_{2}+\alpha_{2} M_{1}^{x_{1}}}{1+M_{1}^{x_{1}}}\right.\right. \\
& \left.\left.-x_{2}(k)\right]\right\} \cdot
\end{aligned}
$$

From (34), similarly to the analysis of (18)-(24), we can finally obtain

$$
\begin{aligned}
& \limsup _{k \rightarrow+\infty} \sum_{s=0}^{+\infty} J_{1}(s) x_{1}(k-s) \leq \limsup _{k \rightarrow+\infty} x_{1}(k) \\
& \leq \frac{K_{1}+\alpha_{1} M_{1}^{x_{2}}}{1+M_{1}^{x_{2}}} \\
& \limsup _{k \rightarrow+\infty} \sum_{s=0}^{+\infty} J_{2}(s) x_{2}(k-s) \leq \limsup _{k \rightarrow+\infty} x_{2}(k) \\
& \leq \frac{K_{2}+\alpha_{2} M_{1}^{x_{1}}}{1+M_{1}^{x_{1}}} .
\end{aligned}
$$

For the above $\varepsilon>0$, it follows from (35) that there exists an integer $k_{3}>k_{2}$ such that, for all $k>k_{3}$,

$$
\begin{array}{r}
x_{1}(k)<\frac{K_{1}+\alpha_{1} M_{1}^{x_{2}}}{1+M_{1}^{x_{2}}}+\frac{\varepsilon}{2} \stackrel{\text { def }}{=} M_{2}^{x_{1}}, \\
x_{2}(k)<\frac{K_{2}+\alpha_{2} M_{1}^{x_{1}}}{1+M_{1}^{x_{1}}}+\frac{\varepsilon}{2} \stackrel{\text { def }}{=} M_{2}^{x_{2}} ; \\
\sum_{s=0}^{+\infty} J_{1}(s) x_{1}(k-s)<\frac{K_{1}+\alpha_{1} M_{1}^{x_{2}}}{1+M_{1}^{x_{2}}}+\frac{\varepsilon}{2} \stackrel{\text { def }}{=} M_{2}^{x_{1}}, \\
\sum_{s=0}^{+\infty} J_{2}(s) x_{2}(k-s)<\frac{K_{2}+\alpha_{2} M_{1}^{x_{1}}}{1+M_{1}^{x_{1}}}+\frac{\varepsilon}{2} \stackrel{\text { def }}{=} M_{2}^{x_{2}} .
\end{array}
$$

It then follows from (25), (26), and (36) that

$$
M_{2}^{x_{i}}<M_{1}^{x_{i}}, \quad i=1,2 .
$$

For $k \geq k_{3}$, from the strictly increasing function $g_{i}(x)=\left(K_{i}+\right.$ $\left.\alpha_{i} x\right) /(1+x), \alpha_{i}>K_{i}, i=1,2$, and (33), we can obtain

$$
\begin{aligned}
& x_{1}(k+1) \\
& \quad \geq x_{1}(k) \exp \left\{r_{1}\left[\frac{K_{1}+\alpha_{1} m_{1}^{x_{2}}}{1+m_{1}^{x_{2}}}-x_{1}(k)\right]\right\}, \\
& x_{2}(k+1) \\
& \quad \geq x_{2}(k) \exp \left\{r_{2}\left[\frac{K_{2}+\alpha_{2} m_{1}^{x_{1}}}{1+m_{1}^{x_{1}}}-x_{2}(k)\right]\right\} .
\end{aligned}
$$

From (38), similar to the analysis of (27)-(32), we can obtain

$$
\begin{aligned}
& V_{1}=\liminf _{k \rightarrow+\infty} x_{1}(k) \geq \frac{K_{1}+\alpha_{1} m_{1}^{x_{2}}}{1+m_{1}^{x_{2}}}, \\
& \liminf _{k \rightarrow+\infty} \sum_{s=0}^{+\infty} J_{1}(s) x_{1}(k-s) \geq \frac{K_{1}+\alpha_{1} m_{1}^{x_{2}}}{1+m_{1}^{x_{2}}}, \\
& V_{2}=\liminf _{k \rightarrow+\infty} x_{2}(k) \geq \frac{K_{2}+\alpha_{2} m_{1}^{x_{1}}}{1+m_{1}^{x_{1}}}, \\
& \liminf _{k \rightarrow+\infty} \sum_{s=0}^{+\infty} J_{1}(s) x_{1}(k-s) \geq \frac{K_{2}+\alpha_{2} m_{1}^{x_{1}}}{1+m_{1}^{x_{1}}} .
\end{aligned}
$$

For the above $\varepsilon>0$, it follows from (39) that there is an integer $k_{4}>k_{3}$ such that, for all $k>k_{4}$,

$$
\begin{aligned}
& x_{1}(k)>\frac{K_{1}+\alpha_{1} m_{1}^{x_{2}}}{1+m_{1}^{x_{2}}}-\frac{\varepsilon}{2} \stackrel{\text { def }}{=} m_{2}^{x_{1}}, \\
& x_{2}(k)>\frac{K_{2}+\alpha_{2} m_{1}^{x_{1}}}{1+m_{1}^{x_{1}}}-\frac{\varepsilon}{2} \stackrel{\text { def }}{=} m_{2}^{x_{2}}, \\
& \sum_{s=0}^{+\infty} J_{1}(s) x_{1}(k-s)>\frac{K_{1}+\alpha_{1} m_{1}^{x_{2}}}{1+m_{1}^{x_{2}}}-\frac{\varepsilon}{2} \stackrel{\text { def }}{=} m_{2}^{x_{1}}, \\
& \sum_{s=0}^{+\infty} J_{2}(s) x_{2}(k-s)>\frac{K_{2}+\alpha_{2} m_{1}^{x_{1}}}{1+m_{1}^{x_{1}}}-\frac{\varepsilon}{2} \stackrel{\text { def }}{=} m_{2}^{x_{2}} .
\end{aligned}
$$


Noting that

$$
\begin{aligned}
& \frac{K_{1}+\alpha_{1} m_{1}^{x_{2}}}{1+m_{1}^{x_{2}}}>K_{1}, \\
& \frac{K_{2}+\alpha_{2} m_{1}^{x_{1}}}{1+m_{1}^{x_{1}}}>K_{2}, \\
& \quad i=1,2 .
\end{aligned}
$$

Then from (33) and (40) we have

$$
m_{2}^{x_{i}}>m_{1}^{x_{i}}, \quad i=1,2
$$

Continuing the above steps, we can get four sequences $\left\{M_{k}^{x_{1}}\right\}$, $\left\{M_{k}^{x_{2}}\right\},\left\{m_{k}^{x_{1}}\right\}$, and $\left\{m_{k}^{x_{2}}\right\}$ such that

$$
\begin{aligned}
& M_{k}^{x_{1}}=\frac{K_{1}+\alpha_{1} M_{k-1}^{x_{2}}}{1+M_{k-1}^{x_{2}}}+\frac{\varepsilon}{k}, \\
& M_{k}^{x_{2}}=\frac{K_{2}+\alpha_{2} M_{k-1}^{x_{1}}}{1+M_{k-1}^{x_{1}}}+\frac{\varepsilon}{k} ; \\
& m_{k}^{x_{1}}=\frac{K_{1}+\alpha_{1} m_{k-1}^{x_{2}}}{1+m_{k-1}^{x_{2}}}-\frac{\varepsilon}{k}, \\
& m_{k}^{x_{2}}=\frac{K_{2}+\alpha_{2} m_{k-1}^{x_{1}}}{1+m_{k-1}^{x_{1}}}-\frac{\varepsilon}{k} .
\end{aligned}
$$

Clearly, we have

$$
\begin{aligned}
& m_{k}^{x_{1}}<V_{1} \leq U_{1}<M_{k}^{x_{1}}, \\
& m_{k}^{x_{2}}<V_{2} \leq U_{2}<M_{k}^{x_{2}}, \\
& \quad k=0,1,2, \ldots .
\end{aligned}
$$

Now, we will prove $\left\{M_{k}^{x_{i}}\right\}(i=1,2)$ is monotonically decreasing and $\left\{m_{k}^{x_{i}}\right\}(i=1,2)$ is monotonically increasing by means of inductive method.

First of all, from (37) and (42) we have $M_{2}^{x_{i}}<M_{1}^{x_{i}}, m_{2}^{x_{i}}>$ $m_{1}^{x_{i}}(i=1,2)$. For $k \geq 2$, we assume that $M_{k}^{x_{i}}<M_{k-1}^{x_{i}}$ and $m_{k}^{x_{i}}>m_{k-1}^{x_{i}}, i=1,2$, holds, and then from the strictly increasing of function $g_{i}(x)=\left(K_{i}+\alpha_{i} x\right) /(1+x), i=1,2$, it immediately follows that

$$
\begin{aligned}
M_{k+1}^{x_{1}} & =\frac{K_{1}+\alpha_{1} M_{k}^{x_{2}}}{1+M_{k}^{x_{2}}}+\frac{\varepsilon}{k+1}<\frac{K_{1}+\alpha_{1} M_{k-1}^{x_{2}}}{1+M_{k-1}^{x_{2}}}+\frac{\varepsilon}{k} \\
& =M_{k}^{x_{1}} ; \\
M_{k+1}^{x_{2}} & =\frac{K_{2}+\alpha_{2} M_{k}^{x_{1}}}{1+M_{k}^{x_{1}}}+\frac{\varepsilon}{k+1}<\frac{K_{2}+\alpha_{2} M_{k-1}^{x_{1}}}{1+M_{k-1}^{x_{1}}}+\frac{\varepsilon}{k} \\
& =M_{k}^{x_{2}},
\end{aligned}
$$

$$
\begin{aligned}
m_{k+1}^{x_{1}} & =\frac{K_{1}+\alpha_{1} m_{k}^{x_{2}}}{1+m_{k}^{x_{2}}}-\frac{\varepsilon}{k+1}>\frac{K_{1}+\alpha_{1} m_{k-1}^{x_{2}}}{1+m_{k-1}^{x_{2}}}-\frac{\varepsilon}{k} \\
& =M_{k}^{x_{1}} ; \\
m_{k+1}^{x_{2}} & =\frac{K_{2}+\alpha_{2} m_{k}^{x_{1}}}{1+m_{k}^{x_{1}}}-\frac{\varepsilon}{k+1}>\frac{K_{2}+\alpha_{2} m_{k-1}^{x_{1}}}{1+m_{k-1}^{x_{1}}}-\frac{\varepsilon}{k} \\
& =M_{k}^{x_{2}} .
\end{aligned}
$$

Equations of (45) show that $\left\{M_{k}^{x_{i}}\right\}(i=1,2)$ is monotonically decreasing and $\left\{m_{k}^{x_{i}}\right\}(i=1,2)$ is monotonically increasing. Consequently, $\lim _{k \rightarrow+\infty}\left\{M_{k}^{x_{i}}\right\}$ and $\lim _{k \rightarrow+\infty}\left\{m_{k}^{x_{i}}\right\}(i=1,2)$ both exist. Let

$$
\begin{aligned}
& \lim _{k \rightarrow+\infty} M_{k}^{x_{i}}=\bar{X}_{i}, \\
& \lim _{k \rightarrow+\infty} m_{k}^{x_{i}}=\underline{X}_{i}, \\
& \\
& i=1,2 .
\end{aligned}
$$

From (43), we have

$$
\begin{aligned}
& \bar{X}_{1}=\frac{K_{1}+\alpha_{1} \bar{X}_{2}}{1+\bar{X}_{2}} ; \\
& \bar{X}_{2}=\frac{K_{2}+\alpha_{2} \bar{X}_{1}}{1+\bar{X}_{1}} ; \\
& \underline{X}_{1}=\frac{K_{1}+\alpha_{1} \underline{X}_{2}}{1+\underline{X}_{2}} ; \\
& \underline{X}_{2}=\frac{K_{2}+\alpha_{2} \underline{X}_{1}}{1+\underline{X}_{1}} ;
\end{aligned}
$$

Here, (47) shows that $\left(\bar{X}_{1}, \bar{X}_{2}\right)$ and $\left(\underline{X}_{1}, \underline{X}_{2}\right)$ are all solutions of system (7). However, system (7) has unique positive solution $\left(x_{1}^{*}, x_{2}^{*}\right)$. Therefore

$$
U_{i}=V_{i}=\lim _{k \rightarrow+\infty} x_{i}(k)=x_{i}^{*}, \quad i=1,2 ;
$$

that is, $E_{+}\left(x_{1}^{*}, x_{2}^{*}\right)$ is globally attractive. The proof of the theorem is completed.

\section{Examples}

In this section we shall give an example to illustrate the feasibility of the main result.

Example 1. Consider the following example:

$$
\begin{aligned}
& x_{1}(k+1)=x_{1}(k) \\
& \cdot \exp \left\{3 \left[\frac{0.2+0.3 \sum_{s=0}^{\infty}((e-1) / e) e^{-s} x_{2}(n-s)}{1+\sum_{s=0}^{\infty}((e-1) / e) e^{-s} x_{2}(n-s)}\right.\right. \\
& \left.\left.\quad-x_{1}(k)\right]\right\},
\end{aligned}
$$




$$
\begin{aligned}
& x_{2}(k+1)=x_{2}(k) \\
& \cdot \exp \left\{0 . 5 \left[\frac{0.5+1.5 \sum_{s=0}^{\infty}((e-1) / e) e^{-s} x_{1}(n-s)}{1+\sum_{s=0}^{\infty}((e-1) / e) e^{-s} x_{1}(n-s)}\right.\right. \\
& \left.\left.-x_{2}(k)\right]\right\} .
\end{aligned}
$$

Corresponding to system (1), we have $r_{1}=3, K_{1}=0.2, \alpha_{1}=$ $0.3, r_{2}=0.5, K_{2}=0.5, \alpha_{2}=1.5$, and hence

$$
\alpha_{i}>K_{i}, \quad i=1,2
$$

Also,

$$
\begin{aligned}
& r_{1} \alpha_{1}=0.9<1, \\
& r_{2} \alpha_{2}=0.75<1 .
\end{aligned}
$$

Hence, all the conditions of Theorem 1 hold, and it follows from Theorem 1 that system (49) admits a unique globally attractive positive equilibrium. Figure 1 supports this assertion.

Example 2. Consider the following example:

$$
\begin{aligned}
& x_{1}(k+1)=x_{1}(k) \\
& \cdot \exp \left\{5 \left[\frac{0.2+0.3 \sum_{s=0}^{\infty}((e-1) / e) e^{-s} x_{2}(n-s)}{1+\sum_{s=0}^{\infty}((e-1) / e) e^{-s} x_{2}(n-s)}\right.\right. \\
& \left.\left.-x_{1}(k)\right]\right\}, \\
& x_{2}(k+1)=x_{2}(k) \\
& \cdot \exp \left\{1 \left[\frac{0.5+1.5 \sum_{s=0}^{\infty}((e-1) / e) e^{-s} x_{1}(n-s)}{1+\sum_{s=0}^{\infty}((e-1) / e) e^{-s} x_{1}(n-s)}\right.\right. \\
& \left.\left.-x_{2}(k)\right]\right\} .
\end{aligned}
$$

Corresponding to system (1), we have $r_{1}=5, K_{1}=0.2, \alpha_{1}=$ $0.3, r_{2}=1, K_{2}=0.5, \alpha_{2}=1.5$, and, obviously,

$$
\alpha_{i}>K_{i}, \quad i=1,2 \text {. }
$$

However,

$$
\begin{aligned}
& r_{1} \alpha_{1}=1.5>1, \\
& r_{2} \alpha_{2}=1.5>1 .
\end{aligned}
$$

Hence, condition $\left(\mathrm{H}_{2}\right)$ in Theorem 1 could not be satisfied, and Theorem 1 could not be applied to this example. However, numeric simulation (Figure 2) also shows that system (52) admits a unique globally attractive positive equilibrium.

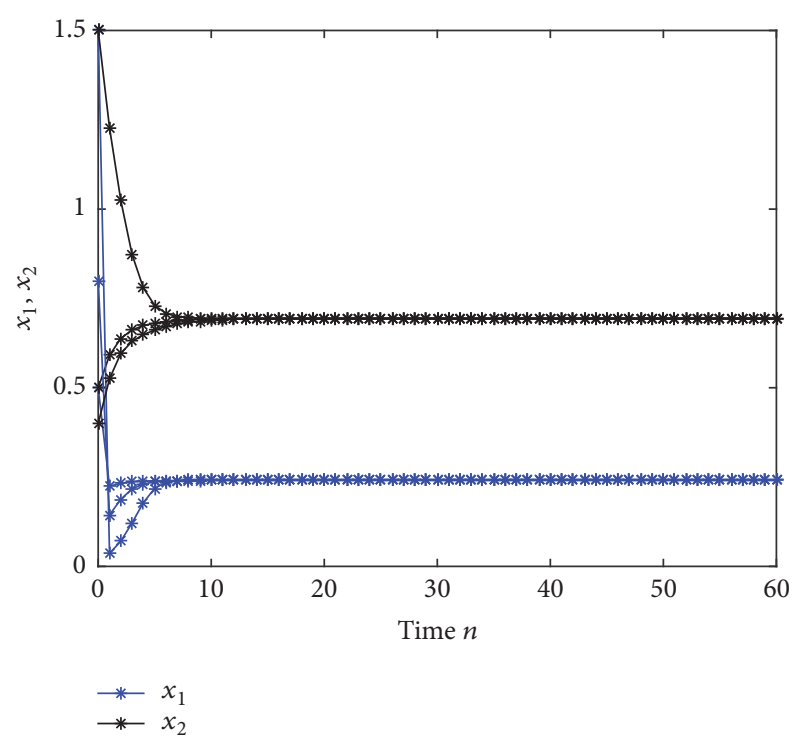

FIGURE 1: Dynamic behaviors of the solution $\left(x_{1}(n), x_{2}(n)\right)$ of system (49), with the initial conditions $\left(x_{1}(s), x_{2}(s)\right)=(0.8,0.4),(0.5,0.5)$, and $(1.5,1.5), s=\cdots,-n,-n+1, \ldots,-1,0$, respectively.

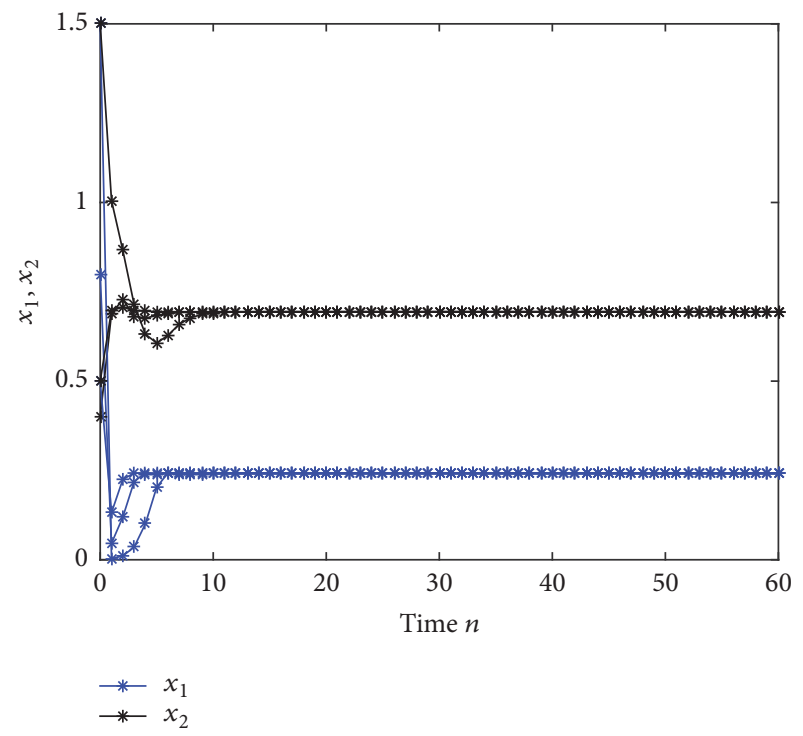

FIGURE 2: Dynamic behaviors of the solution $\left(x_{1}(n), x_{2}(n)\right)$ of system (52), with the initial conditions $\left(x_{1}(s), x_{2}(s)\right)=(0.8,0.4),(0.5,0.5)$, and $(1.5,1.5), s=\cdots,-n,-n+1, \ldots,-1,0$, respectively.

\section{Discussion}

In [6], Yang et al. proposed system (6); under the assumption $\alpha_{i}>K_{i}, i=1,2$, they showed that if $r_{i} \alpha_{i} \leq 1$, then the mutualism model admits a unique globally asymptotically stable positive equilibrium.

In this paper, we try to incorporate the infinite deviating arguments, and, by developing the analysis technique of Yang et al. [6] and using the difference inequality of Chen [7], we also obtain the sufficient conditions which ensure the global attractivity of the positive equilibrium. Example 1 shows the feasibility of our main result. 
Since condition $\left(\mathrm{H}_{2}\right)$ is the most important restriction on the coefficients of the system, one interesting issue is whether the result of Theorem 3 could hold if $\left(\mathrm{H}_{2}\right)$ is not satisfied. Example 2 shows that our result (Theorem 3 ) still have room to improve. We leave this for future investigation.

\section{Competing Interests}

The authors declare that there is no conflict of interests regarding the publication of this paper.

\section{Acknowledgments}

The research was supported by the Natural Science Foundation of Fujian Province (2015J01019).

\section{References}

[1] Y. Li and G. Xu, "Positive periodic solutions for an integrodifferential model of mutualism," Applied Mathematics Letters, vol. 14, no. 5, pp. 525-530, 2001.

[2] F. Chen and M. You, "Permanence for an integrodifferential model of mutualism," Applied Mathematics and Computation, vol. 186, no. 1, pp. 30-34, 2007.

[3] X. Xie, F. Chen, K. Yang, and Y. Xue, "Global attractivity of an integrodifferential model of mutualism," Abstract and Applied Analysis, vol. 2014, Article ID 928726, 6 pages, 2014.

[4] X. Li and W. Yang, "Permanence of a discrete model of mutualism with infinite deviating arguments," Discrete Dynamics in Nature and Society, vol. 2010, Article ID 931798, 7 pages, 2010.

[5] Z. Li, "Permanence for the discrete mutualism model with delays," Journal of Mathematics Study, vol. 43, no. 1, pp. 51-54, 2010.

[6] K. Yang, X. Xie, and F. Chen, "Global stability of a discrete mutualism model," Abstract and Applied Analysis, vol. 2014, Article ID 709124, 7 pages, 2014.

[7] B. G. Chen, "Global attractivity of a discrete competition model," Advances in Difference Equations, vol. 2016, article 273, 2016.

[8] Y. Li and H. Zhang, "Existence of periodic solutions for a periodic mutualism model on time scales," Journal of Mathematical Analysis and Applications, vol. 343, no. 2, pp. 818-825, 2008.

[9] Y. Li, "On a periodic mutualism model," The ANZIAM Journal., vol. 42, no. 4, pp. 569-580, 2001.

[10] Y. Li and T. Zhang, "Permanence of a discrete n-species cooperation system with time-varying delays and feedback controls," Mathematical \& Computer Modelling, vol. 53, no. 5-6, pp. 13201330, 2011.

[11] H. Zhang, Y. Li, B. Jing, and W. Zhao, "Global stability of almost periodic solution of multispecies mutualism system with time delays and impulsive effects," Applied Mathematics and Computation, vol. 232, pp. 1138-1150, 2014.

[12] F. Chen, "Permanence for the discrete mutualism model with time delays," Mathematical and Computer Modelling, vol. 47, no. 3-4, pp. 431-435, 2008.

[13] H. Zhang, F. Feng, B. Jing, and Y. Li, "Almost periodic solution of a multispecies discrete mutualism system with feedback controls," Discrete Dynamics in Nature and Society, vol. 2015, Article ID 268378, 14 pages, 2015.
[14] Z. Liu, J. Wu, R. Tan, and Y. Chen, "Modeling and analysis of a periodic delayed two-species model of facultative mutualism," Applied Mathematics and Computation, vol. 217, no. 2, pp. 893903, 2010.

[15] F. D. Chen and X. D. Xie, Study on the Dynamic Behaviors of Cooperation Population Modeling, Science Press, Beijing, China, 2014.

[16] L. Chen, L. Chen, and Z. Li, "Permanence of a delayed discrete mutualism model with feedback controls," Mathematical and Computer Modelling, vol. 50, no. 7-8, pp. 1083-1089, 2009.

[17] L. Chen and X. Xie, "Permanence of an N-species cooperation system with continuous time delays and feedback controls," Nonlinear Analysis: Real World Applications, vol. 12, no. 1, pp. 34-38, 2011.

[18] L. Chen, X. Xie, and L. Chen, "Feedback control variables have no influence on the permanence of a discrete $N$-species cooperation system," Discrete Dynamics in Nature and Society, vol. 2009, Article ID 306425, 10 pages, 2009.

[19] X. Xie, F. Chen, and Y. Xue, "Note on the stability property of a cooperative system incorporating harvesting," Discrete Dynamics in Nature and Society. An International Multidisciplinary Research and Review Journal, vol. 2014, Article ID 327823, 5 pages, 2014.

[20] Y. Liao and T. Zhang, "Almost periodic solutions of a discrete mutualism model with variable delays," Discrete Dynamics in Nature \& Society, vol. 2012, Article ID 742102, 27 pages, 2012.

[21] K. Yang, Z. Miao, F. Chen, and X. Xie, "Influence of single feedback control variable on an autonomous Holling-II type cooperative system," Journal of Mathematical Analysis and Applications, vol. 435, no. 1, pp. 874-888, 2016.

[22] F. D. Chen, X. D. Xie, and X. F. Chen, "Dynamic behaviors of a stage-structured cooperation model," Communications in Mathematical Biology and Neuro-Science, vol. 2015, article 4, 2015.

[23] D. Mukherjee, "Permanence and global attractivity for facultative mutualism system with delay," Mathematical Methods in the Applied Sciences, vol. 26, no. 1, pp. 1-9, 2003.

[24] B. Chen, "Permanence for the discrete competition model with infinite deviating arguments," Discrete Dynamics in Nature and Society, vol. 2016, Article ID 1686973, 5 pages, 2016.

[25] G. Chen and Z. Teng, "On the stability in a discrete two-species competition system," Journal of Applied Mathematics and Computing, vol. 38, no. 1-2, pp. 25-39, 2012.

[26] W. Guo-ying, "A high accuracy difference scheme for the singular perturbation problem of the second-order linear ordinary differential equation in conservation form," Applied Mathematics and Mechanics, vol. 10, no. 5, pp. 465-470, 1989.

[27] F. D. Chen, "Permanence in a discrete Lotka-Volterra competition model with deviating arguments," Nonlinear Analysis: Real World Applications, vol. 9, no. 5, pp. 2150-2155, 2008. 


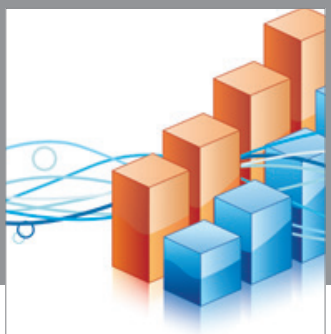

Advances in

Operations Research

vatem alat4

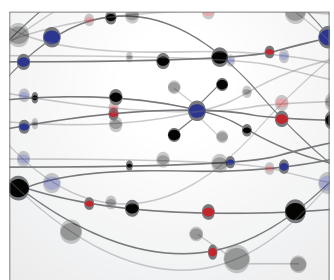

\section{The Scientific} World Journal
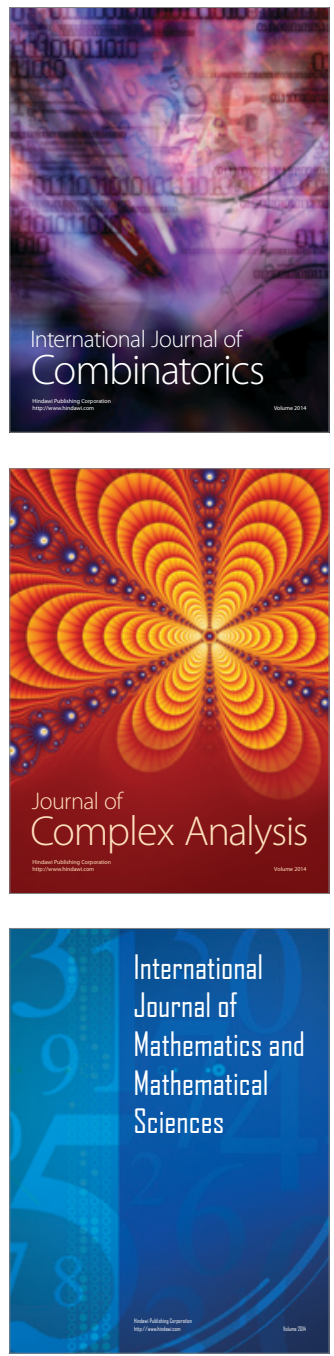
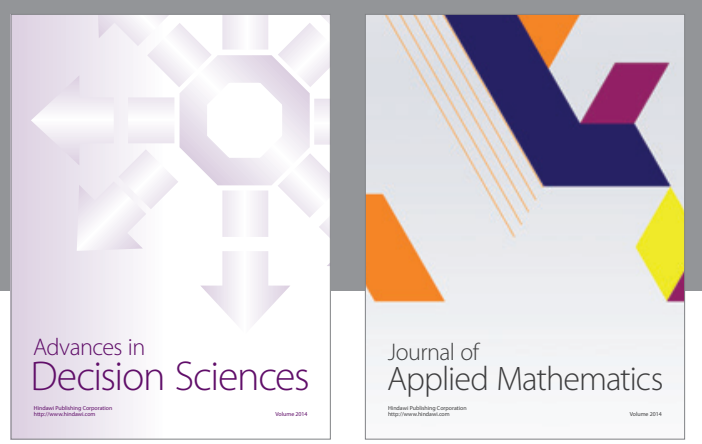

Algebra

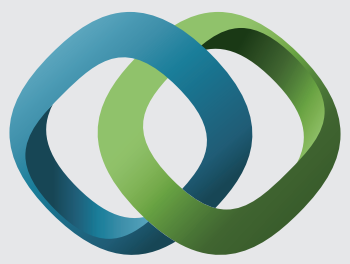

\section{Hindawi}

Submit your manuscripts at

https://www.hindawi.com
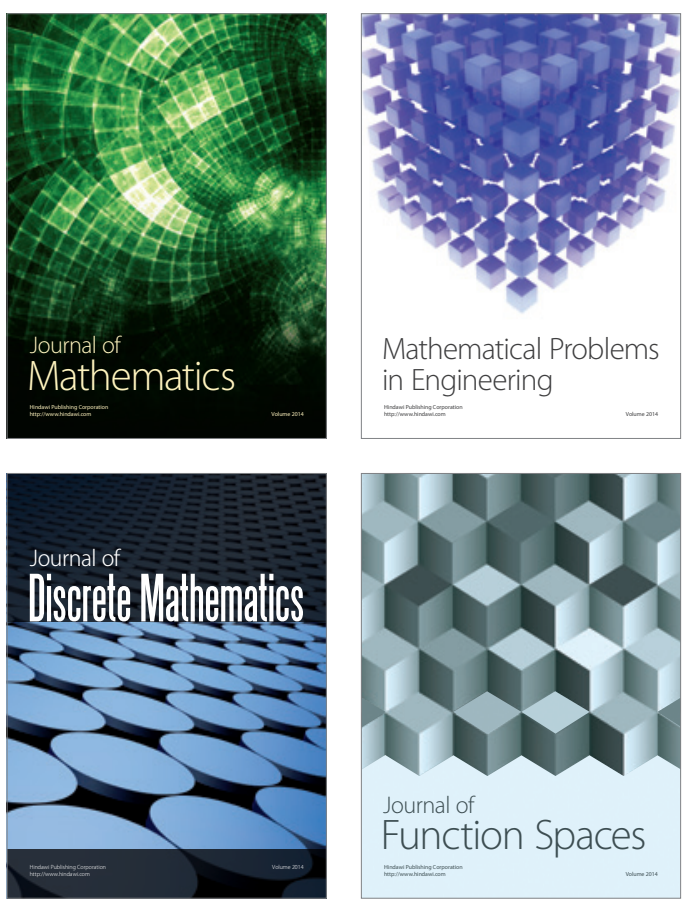

Mathematical Problems in Engineering
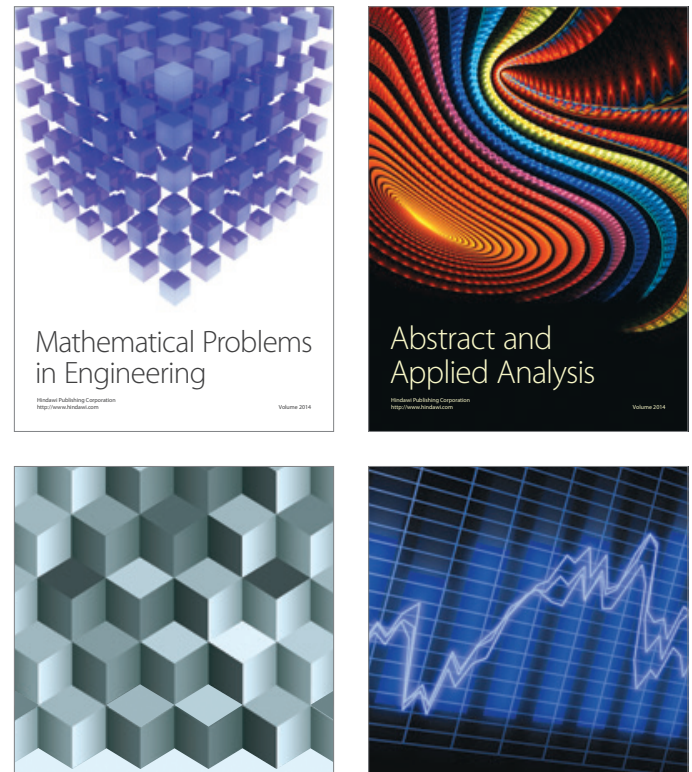

Journal of

Function Spaces

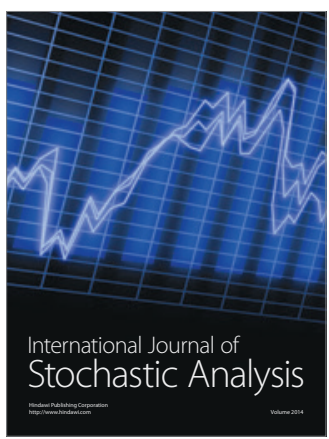

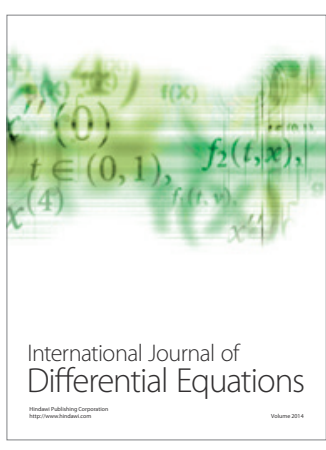
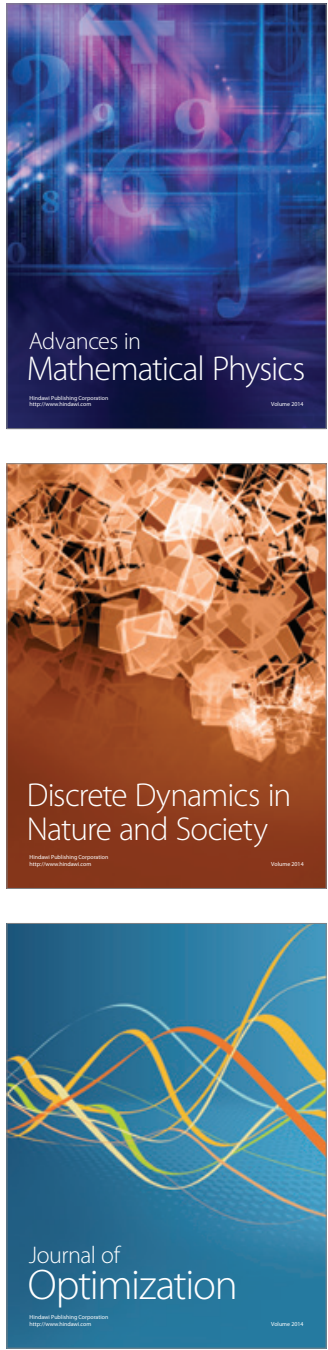